\title{
FUNDAMENTAL SOLUTIONS IN COMPUTATIONAL FLUID DYNAMICS
}

\author{
LEOPOLD ŠKERGET ${ }^{1,2}$, ANTÓNIO TADEU ${ }^{2,3}$ \& JURE RAVNIK ${ }^{4}$ \\ ${ }^{1}$ Wessex Institute of Technology, UK \\ ${ }^{2}$ ITeCons - Institute for Research and Technological Development in Construction, \\ Energy, Environment and Sustainability, Portugal \\ ${ }^{3}$ ADAI - LAETA, Department of Civil Engineering, University of Coimbra, Portugal \\ ${ }^{4}$ Faculty of Mechanical Engineering, University of Maribor, Slovenia
}

\begin{abstract}
With the boundary element method (BEM), the velocity-vorticity formulation is introduced and the overall Navier-Stokes problem is partitioned into the kinetic and kinematic parts. For a general viscous flow, the kinetics is formulated as a differential nonlinear vorticity diffusion-convective transport equation, whilst the kinematics of the fluid flow computation is governed by the BiotSavart integral representation. This work presents an overview of the numerical simulation of transport phenomena in fluid flow using a different type of Green's fundamental solutions in the context of BEM. The kinetic diffusion-convective partial differential equations (PDEs) represent, respectively, mixed elliptic-hyperbolic or parabolic-hyperbolic types of PDEs, governing the steady or time dependent transport phenomena in fluid flow, e.g. transfer of heat energy, momentum, vorticity, etc. Applying the singular integral representations has important numerical and physical aspects as a consequence of the fundamental solutions applied. The solution algorithm is based on improved macro-elements concept using mixed-boundary elements. The numerical model uses quadratic approximation for all field functions and linear approximation of the fluxes over space and constant approximation over time for all field functions.

Keywords: computational fluid dynamics, boundary element method (BEM), macro-element model, Green's fundamental solutions, high Rayleigh number, natural convection.
\end{abstract}

\section{INTRODUCTION}

The current literature contains a variety of differing and competing numerical models for solving transport problems governed by the Navier-Stokes equations. A number of the numerical models can be categorised according to the mathematical formulations they use, e.g. finite difference/finite volume (FVM), finite element (FEM) and boundary element (BEM) models [1], [7], [10], [13], [15], [18].

The key problem with all numerical models is how to satisfactorily solve the kinetics of the transport problems governed by the diffusion-convection equations. The characteristics of the diffusion-convection equations vary considerably from point to point in the flow field due to the local Reynolds or Peclet number values that physically represent the relationship between the diffusion and convection of individual flow quantities. The mixed elliptic-parabolic-hyperbolic character of the underlying equations makes the numerical fluid dynamics more difficult than numerically solving the transport phenomena in solids. This is especially true for flows characterised by high Reynolds or Peclet numbers, when convection becomes dominant at the expense of diffusion, or when the hyperbolic character of the equation predominates in its ellipticity/parabolicity.

The diffusion-convection character of the kinetic transport equations, means that when the hyperbolicity prevails in the ellipticity/parabolicity of the PDE, the convection dominated flows are subjected to numerical instability. Therefore, when using a coarse mesh any given numerical solution model which does not take the multiscale nature of a transport problem into account may generate unstable results. To suppress such instabilities which are known in 
all domain type numerical models, such as FVM and FEM, first or higher order upwinding schemes, the residual free bubble (RFB) method etc., have to be considered to stabilise the numerical solution. Although this increases the stability of the numerical scheme, at the same time it also introduces artificial diffusivity, resulting in a non-physical numerical solution.

Since the boundary integral representations are based on the use of an appropriate fundamental solution, the free space Green's function, which incorporates more or less of the physics of the transport phenomenon, such as accumulation, diffusion, convection and generation of the field function, the stable and accurate description of different flow components with diverse length scales can be accommodated and treated much more accurately in a physically and mathematically justified manner.

\section{GOVERNING CONSERVATION EQUATIONS}

In velocity-vorticity formulation the noncompressible viscous fluid motion computation procedure can be split into its kinetics and kinematics [1], [5], [17], [20], [24], [25]. The kinematics deals with the relationship and restriction within the velocity field $v_{i}\left(r_{j}, t\right)$ at any given instant in time and the vorticity field $\omega_{i}\left(r_{j}, t\right)$ at the same instant. For the twodimensional plane motion the kinematics is given by the following vector elliptic Poisson equation for the velocity vector,

$$
\frac{\partial^{2} v_{i}}{\partial x_{j} \partial x_{j}}+e_{i j} \frac{\partial \omega}{\partial x_{j}}=0
$$

where $e_{i j}(i, j=1,2)$ is the permutation unit symbol $\left(e_{12}=+1, e_{21}=-1, e_{11}=e_{22}=0\right)$. For the known vorticity the corresponding velocity vector can be determined by solving eqn (1), provide that appropriate boundary conditions for the velocity are prescribed, i.e. normal and tangential component of the velocity vector. The kinetic aspects of the fluid motion and energy transfer are governed by the scalar vorticity and temperature diffusionconvection transport equations,

$$
\begin{gathered}
\frac{\partial \omega}{\partial t}+\frac{\partial v_{j} \omega}{\partial x_{j}}=\nu_{o} \frac{\partial^{2} \omega}{\partial x_{j} \partial x_{j}}-\frac{1}{\rho_{o}} e_{i j} \frac{\partial}{\partial x_{j}}\left(\rho g_{i}+f_{i}^{n}\right), \\
\frac{\partial T}{\partial t}+\frac{\partial v_{j} T}{\partial x_{j}}=a_{o} \frac{\partial^{2} T}{\partial x_{j} \partial x_{j}}+\frac{1}{c_{o}}\left(I_{T}+S^{n}\right) .
\end{gathered}
$$

These describe the redistribution of the vorticity/temperature in the fluid domain by diffusion and convection, whilst the nonhomogeneous nonlinear terms $f_{i}^{n}$ and $S^{n}$, act as an additional source or strengthen terms because of the nonlinear transport properties. The notations $\nu$, $\rho, c$ and $a=k / c$ stand for the kinematic viscosity, mass density, specific isobaric heat per unit volume and thermal diffusivity, where $k$ is the heat conductivity, $g_{i}$ is the gravitational acceleration vector, and $I_{T}$ is the heat source per unit volume.

\section{GENERAL SCALAR TRANSPORT DIFFUSION-CONVECTIVE EQUATION}

Let us consider a general unsteady state nonlinear diffusion-convective equation describing the time dependent transfer of an arbitrary scalar field function $u(\vec{r}, t)$, which can be equated to vorticity, temperature, concentration, etc., in an isotropic solenoidal fluid flow, e.g. divergence free, defined in a solution space $\mathcal{R}(s, t)=\Omega(s) \times \mathcal{I}\left(t_{o}, t\right)$ representing the product of the domain $\Omega(s)$ bounded by the boundary $\Gamma(S)$ and the time interval $\mathcal{I}\left(t_{o}, t\right)$, e.g. given in indicial notation form for a right-handed Cartesian coordinate system

$$
\frac{\partial}{\partial x_{j}}\left(k \frac{\partial u}{\partial x_{j}}\right)-c\left(\frac{\partial v_{j} u}{\partial x_{j}}+\frac{\partial u}{\partial t}\right)+I_{u}=0 \quad \text { in } \mathcal{R},
$$


where $\vec{v}(\vec{r}, t)$ is the local instantaneous velocity field. The material properties of the media, e.g. capacity per unit volume $c=c(\vec{r}, u)$ and conductivity $k=k(\vec{r}, u)$, are, as a rule, a monotonic field function and space dependent relation, while $I_{u}(\vec{r}, u)$ stands for a source or sink term.

Eqn (4) represents a parabolic initial-boundary values problem, thus some boundary and initial conditions have to be specified to complete the mathematical description of the problem, e.g. Dirichlet, Neumann or Cauchy type boundary conditions have to be prescribed on the part of the boundary $\Gamma=\Gamma_{1}+\Gamma_{2}+\Gamma_{3}$ defined by the outward unit normal vector $n_{i}(S)$,

$$
\begin{aligned}
u=\bar{u} \quad \text { on } \quad \mathcal{R}_{\Gamma_{1}}=\Gamma_{1} \times \mathcal{I}, \\
\frac{\partial u}{\partial n}=\bar{q} \quad \text { on } \quad \mathcal{R}_{\Gamma_{2}}=\Gamma_{2} \times \mathcal{I}, \\
\frac{\partial u}{\partial n}=-\frac{\alpha}{k}\left(u-u_{f}\right) \quad \text { on } \quad \mathcal{R}_{\Gamma_{3}}=\Gamma_{3} \times \mathcal{I},
\end{aligned}
$$

where $\alpha$ is a transfer coefficient between the fluid flow surface and the surrounding ambient at the reference value $u_{f}$, whilst the initial conditions are

$$
u=u_{o} \quad \text { in } \Omega \text { at } t=t_{o} .
$$

The material properties and the velocity field can be given as the sum of a homogeneous constant dominant and the variable part over each macroelement, e.g.,

$$
c=c_{o}+\widetilde{c}, \quad k=k_{o}+\widetilde{k}, \quad v_{i}=v_{i o}+\widetilde{v_{i}} \quad \text { and } \quad a_{o}=\frac{k_{o}}{c_{o}},
$$

enabling eqn (4) to be split into the homogeneous linear and nonhomogeneous nonlinear part, thus,

$$
a_{o} \frac{\partial^{2} u}{\partial x_{j} \partial x_{j}}-\left(\frac{\partial v_{j o} u}{\partial x_{j}}+\frac{\partial u}{\partial t}\right)+\frac{\partial \gamma_{j}}{\partial x_{j}}+\gamma=0 .
$$

The nonlinear pseudo body force terms $\gamma_{j}$ and $\gamma$ include the perturbed diffusion and convection flux, nonlinearities in transport properties, source term, and nonlinear accumulation contribution

$$
\gamma_{j}=\frac{1}{c_{o}}\left(\widetilde{k} \frac{\partial u}{\partial x_{j}}-c v_{j} u+c_{o} v_{j o} u\right), \quad \gamma=\frac{1}{c_{o}}\left(v_{j} u \frac{\partial c}{\partial x_{j}}-\widetilde{c} \frac{\partial u}{\partial t}+I_{u}\right) .
$$

Eqn (8) incorporates the characteristic physics of the transport phenomenon in fluid flow such as accumulation, diffusion, convection, whilst the nonlinear source terms act as additional generation and strengthening of the conservation field function.

Different Green's fundamental solutions can now be applied to capture more or less of the physics of the transport phenomena [3], [4], [6], [11], [12], e.g. accumulation, diffusion only, or both processes, that is, diffusion and convection, respectively, resulting in different numerical schemes for the stability and the accuracy. Since the fundamental solutions only consider the linear transport phenomenon, an appropriate selection of a linear differential operator $\mathcal{L}[\cdot]$ is of major importance in establishing a stable and accurate singular integral representation corresponding to the original differential diffusion convective transport equation, which is especially true for the transport phenomena characterised by the high Peclet number values. For example, in the steady state the linear elliptic differential 
operator can capture just the diffusion or both the diffusion and convection fluxes, and the accumulation is zero. In the unsteady state, however, the linear parabolic differential operator can be adjusted for accumulation and diffusion, or for accumulation, diffusion and convection, and the nonhomogeneous terms deal with transport effects which are not captured by the differential operator. Thus, the following general mathematical statement can be formulated:

$$
\mathcal{L}[u]+\frac{\partial b_{j}}{\partial x_{j}}+b=\left\{\begin{array}{l}
a_{o} \frac{\partial^{2} u}{\partial x_{j} \partial x_{j}}+\frac{\partial b_{j}}{\partial x_{j}}+b=0 \\
a_{o} \frac{\partial^{2} u}{\partial x_{j} \partial x_{j}}-\frac{\partial v_{o j} u}{\partial x_{j}}+\frac{\partial b_{j}}{\partial x_{j}}+b=0, \\
a_{o} \frac{\partial^{2} u}{\partial x_{j} \partial x_{j}}-\frac{\partial u}{\partial t}+\frac{\partial b_{j}}{\partial x_{j}}+b=0 \\
a_{o} \frac{\partial^{2} u}{\partial x_{j} \partial x_{j}}-\frac{\partial u}{\partial t}-\frac{\partial v_{o j} u}{\partial x_{j}}+\frac{\partial b_{j}}{\partial x_{j}}+b=0 .
\end{array}\right.
$$

To develop the singular integral representation for eqn (10), it is necessary to know the Green's fundamental solutions $u^{\star}(\cdot)=u^{\star}\left(\xi, s ; t_{F}, t\right)$ satisfying the adjoint equations,

$$
\mathcal{L}^{\star}\left[u^{\star}(\cdot)\right]+\delta(\cdot)=\left\{\begin{array}{l}
\frac{\partial^{2} u^{\star}}{\partial x_{j} \partial x_{j}}+\delta=0, \\
a_{o} \frac{\partial^{2} u^{\star}}{\partial x_{j} \partial x_{j}}+\frac{\partial v_{o j} u^{\star}}{\partial x_{j}}+\delta=0, \\
a_{o} \frac{\partial^{2} u^{\star}}{\partial x_{j} \partial x_{j}}+\frac{\partial u^{\star}}{\partial t}+\delta=0, \\
a_{o} \frac{\partial^{2} u^{\star}}{\partial x_{j} \partial x_{j}}+\frac{\partial u^{\star}}{\partial t}+\frac{\partial v_{o j} u^{\star}}{\partial x_{j}}+\delta=0,
\end{array}\right.
$$

where $\mathcal{L}^{\star}[\cdot]$ denotes the adjoint differential linear operator to $\mathcal{L}[\cdot]$ and $\delta(\cdot)$ is the Dirac delta function, which are given by the following expressions for the two-dimensional plane geometry,

$$
u^{\star}(\cdot)=\left\{\begin{array}{l}
\frac{1}{2 \pi} \ln \left(\frac{1}{r}\right) \\
\frac{1}{2 \pi a_{o}} K_{o}(\mu r) \exp \left(\frac{v_{o j} r_{j}}{2 a_{o}}\right), \quad \mu=\frac{v_{o}}{2 a_{o}}, \\
\frac{1}{4 \pi a_{o} \tau} \exp \left(-\frac{r^{2}}{4 a_{o} \tau}\right), \\
\frac{1}{4 \pi a_{o} \tau} \exp \left(-\frac{r^{2}}{4 a_{o} \tau}-\frac{v_{o}^{2} \tau}{4 a_{o}}+\frac{v_{o j} r_{j}}{2 a_{o}}\right)
\end{array}\right.
$$

whilst the fundamental solutions gradients are given by the formulas

$$
\frac{\partial u^{\star}(\cdot)}{\partial x_{i}}=\left\{\begin{array}{l}
\frac{1}{2 \pi}\left(\frac{r_{i}}{r^{2}}\right), \\
\frac{1}{2 \pi r^{2} a_{o}}\left[\mu r K_{1}(\mu r) r_{i}-\frac{r^{2}}{2 a_{o}} K_{o}(\mu r) v_{o i}\right] \exp \left(\frac{v_{o j} r_{j}}{2 a_{o}}\right), \\
\frac{r_{i}}{8 \pi a_{o}^{2} \tau^{2}} \exp \left(-\frac{r^{2}}{4 a_{o} \tau}\right), \\
\left(\frac{r_{i}}{8 \pi a_{o}^{2} \tau^{2}}-\frac{v_{i}}{8 \pi a_{o}^{2} \tau}\right) \exp \left(-\frac{r^{2}}{4 a_{o} \tau}-\frac{v_{o}^{2} \tau}{4 a_{o}}+\frac{v_{o j} r_{j}}{2 a_{o}}\right) .
\end{array}\right.
$$


The notations $\left(\xi, t_{F}\right)$ and $(s, t)$ represent the source and field points in the solution domain, respectively, $\tau=t_{F}-t$, with $r$ and $v_{o}$ are the modulus of $r_{j}=x_{j}(\xi)-x_{j}(s)$ and $v_{j}$, respectively, whilst the $K_{o}$ and $K_{1}$ are the modified Bessel functions of the second kind.

It is obvious that the elliptic Laplace fundamental solution depends on the geometry capturing just the conduction of the conservation field function, thus it is the simplest one. The advantage is that the influence matrices of the BEM numerical model have to be computed only once, but the convection is not treated in a special manner. In contrast, the elliptic diffusion-convective fundamental solution depends on the velocity field, too. Both the characteristic fluxes of the transport phenomena in fluid flow, namely the diffusion and convective fluxes, are properly addressed; the disadvantage is that the influence matrices have to be computed in each iterative step, which considerably lowers the economics of the BEM model.

Similar conclusions can be stated about the time dependent fundamental solutions. The parabolic diffusion one accurately weights the accumulation and the diffusion of the conservation field function. It depends on the transport properties, geometry and time step and is thus much more complicated than the elliptic Laplace solution. For the constant transport properties, diffusion coefficient, the influence matrices only have to be computed once again. It is also clearly shown from the physical point of view that the most attractive and complete fundamental solution for dealing with general time dependent transport problems in fluid flow characterised by high Peclet number values, is the last one in eqn (12), which is the parabolic diffusion-convective fundamental solution. It depends on the geometry, material properties, velocity field and time step, therefore the accumulation, diffusion and convection of the conservation field function are properly addressed in the weighting of residual statement.

The corresponding boundary-domain integral representation for eqn (4) or eqn (8) can be formulated by applying a weighted residual technique or simply by Green's theorems, resulting in the following integral statement written for a time step $\Delta t=t_{F}-t_{F-1}$

$$
\begin{aligned}
& c(\xi) u\left(\xi, t_{F}\right)+a_{o} \int_{\Gamma} \int_{t_{F-1}}^{t_{F}} u\left(q^{\star}+\frac{1}{a_{o}} v_{o j} n_{j} u^{\star}\right) d t d \Gamma=a_{o} \int_{\Gamma} \int_{t_{F-1}}^{t_{F}} q u^{\star} d t d \Gamma \\
& +\int_{\Gamma} \int_{t_{F-1}}^{t_{F}} \gamma_{j} n_{j} u^{\star} d t d \Gamma-\int_{\Omega} \int_{t_{F-1}}^{t_{F}}\left(\gamma_{j} q_{j}^{\star}-\gamma u^{\star}\right) d t d \Omega+\int_{\Omega} u_{F-1} u_{F-1}^{\star} d \Omega,
\end{aligned}
$$

where $q=q_{j} n_{j}$ and $q^{\star}=q_{j}^{\star} n_{j}$ are the field function or fundamental solution normal fluxes. Assuming constant variation of all field functions within the individual time increment $\Delta t=t_{F}-t_{F-1}$ the time integrals in eqn (14),

$$
U^{\star}=a_{o} \int_{t_{F-1}}^{t_{F}} u^{\star} d t, \quad Q^{\star}=a_{o} \int_{t_{F-1}}^{t_{F}} q^{\star} d t, \quad Q_{j}^{\star}=a_{o} \int_{t_{F-1}}^{t_{F}} q_{j}^{\star} d t,
$$

can be evaluated analytically for the parabolic diffusion fundamental solution or numerically in the case of the parabolic diffusion-convection fundamental solution. However, it should be noted that integration over time can be done analytically, too, for the three-dimensional parabolic diffusion-convection fundamental solution [6], [8], but the expression is pretty extensive, yielding the following general form of integral representation

$$
\begin{gathered}
c(\xi) u\left(\xi, t_{F}\right)+\int_{\Gamma} u\left(Q^{\star}+\frac{1}{a_{o}} v_{o j} n_{j} U^{\star}\right) d \Gamma=\int_{\Gamma} q U^{\star} d \Gamma \\
+\frac{1}{a_{o}} \int_{\Gamma} \gamma_{j} n_{j} U^{\star} d \Gamma-\frac{1}{a_{o}} \int_{\Omega}\left(\gamma_{j} Q_{j}^{\star}-\gamma U^{\star}\right) d \Omega+\int_{\Omega} u_{F-1} u_{F-1}^{\star} d \Omega .
\end{gathered}
$$


It is obvious, that for the steady state problems the influence of the initial conditions captured by the last domain integral vanishes, and the elliptic fundamental solutions should be applied. Also, for the diffusion type fundamental solutions, the convective flux for the dominant velocity $v_{o}$ is identical to zero. It is also clear that the application of the diffusion-convection fundamental solutions, especially the parabolic one, is much more expensive in computational time compared with the diffusion type of fundamental solution. Therefore, a compromise between stability and computational time cost can be considered when solving diffusionconvection transport phenomena, for example in such a way that for the macroelements characterised by cell Peclet number values lower than a prescribed critical value, e.g. $P e_{c e l l}<P e_{c r}$, the diffusion type fundamental solutions are applied.

\section{THERMALLY DRIVEN CAVITY FLOW}

To evaluate the proposed numerical algorithm, the standard pure buoyancy driven natural convection is considered. The problem has been proposed by Davis [2] as a standard example for comparing different numerical techniques in computational fluid mechanics. The motion is caused by the buoyancy force caused when the uniformly heated left wall is at the temperature $T_{h}=+0.5$, while the right wall is uniformly cooled to the temperature $T c=-0.5$, and the rest of the boundary is adiabatic.

Different nonuniform macro-element meshes were used based on continuous quadratic approximation of all field functions and discontinuous linear approximation of the corresponding normal derivatives over the boundary elements: (a) $M=10 \times 10$, (b) $M=$ $20 \times 20$, (c) $M=40 \times 40$. The meshes were compressed in the normal wall direction in both coordinates with the geometrical series having the ratio between the longest and shortest elements $R=10$. The motion is analysed for Rayleigh number values in the range of $10^{3} \leq R a \leq 10^{9}$ for Prandtl number value $\operatorname{Pr}=0.71$. Table 1 shows the comparison of the computed average Nusselt number values with values of benchmark solution [2], [9], [21], [23]. We can observe very good agreement of BEM results with the benchmark solutions, although rather coarse BEM numerical models were applied.

\section{CONCLUSIONS}

In this work an improved numerical solution algorithm based on BEM numerical model has been discussed. The macroelement concept is based on a continuous quadratic approximation of all field functions and the discontinuous linear approximation of corresponding normal derivatives.

Integral representations of the diffusion-convection PDE have been discussed for four different fundamental solutions, which capture more or less physics of a general transport

Table 1: Nusselt number values for different Rayleigh number values.

\begin{tabular}{|c||c|c|c|c|c|c|c|}
\hline $\mathrm{Ra}$ & $10^{3}$ & $10^{4}$ & $10^{5}$ & $10^{6}$ & $10^{7}$ & $10^{8}$ & $10^{9}$ \\
\hline \hline $10 \times 10$ & 1.116 & 2.242 & 4.521 & - & - & - & - \\
\hline $20 \times 20$ & 1.117 & 2.244 & 4.521 & 8.828 & 16.548 & - & - \\
\hline $40 \times 40$ & 1.118 & 2.245 & 4.522 & 8.826 & 16.529 & 30.2 & $55.9-56.2$ \\
\hline$[2],[9],[21],[23]$ & 1.118 & 2.243 & 4.519 & 8.825 & 16.523 & 30.225 & $57.35-58.1$ \\
\hline
\end{tabular}


phenomena. The parabolic diffusion-convective fundamental solution, especially, shows its superiority over all others; however, its applicability is limited by the critical Peclet number value, due to high computation cost. Therefore, it is applied only in the macro-elements with the dominant convection flux, $P e_{\text {cell }}>P e_{c r}$.

\section{ACKNOWLEDGEMENTS}

This work has been supported by the project POCI-01-0247-FEDER-017889 (Inovwall), funded by Portugal 2020 through the Operational Programme for Competitiveness Factors (COMPETE 2020).

\section{REFERENCES}

[1] Wu, J.C., Problems of general viscous flows. Developments in Boundary Element Methods vol. 2, Applied Science Publishers: London, pp. 69-109, 1982.

[2] Davis, G. De Vahl, Natural convection of air in a square cavity: a benchmark solution. Internat. J. Numer. Methods Fluids, 3(2), pp. 249-264, 1983.

[3] Škerget, L. and Brebbia, C.A., Diffusion-convection problems using boundary elements. Adv. Water Resources, 7, pp. 50-57, 1984.

[4] Okamoto, N., Analysis of convective diffusion problem with first-order chemical reaction by boundary element method. Internat. J. Numer. Methods Fluids, 8, pp. 55-64, 1988.

[5] Škerget, L., Alujevič, A., Brebbia, C.A. \& Kuhn, G., Natural and forced convection simulation using the velocity-vorticity approach. Topics in Boundary Element Research vol. 5, Springer: Berlin, pp. 49-86, 1989.

[6] Kawamura, R. \& Fukuma, M., Analysis of three-dimensional transient advection diffusion by boundary element method. Simulation Soc., 9, pp. 160-165, 1989.

[7] Hirsch, C., Numerical computation of internal and external flows. Computational Methods for Inviscid and Viscous Flows vol. 2, John Wiley and Sons: New York, Chichester, Brisbane, Toronto, Singapore, 1990.

[8] Kawamura,R. \& Oikawa, Y., The study of three-dimensional magnetic problem using direct integration on time of the fundamental solution. Boundary Element Technology vol. VII, Springer: The Netherlands, pp. 349-357, 1991.

[9] Le Quere, P., Accurate solutions to the square thermally driven cavity at high Rayleigh number. Comput. \& Fluids, 20(1), pp. 29-41, 1991.

[10] Reddy, J.N. \& Gartling, D.K., The Finite Element Method in Heat Transfer and Fluid Dynamics, CRC Press: Boca Raton, 1994.

[11] Žagar, I. \& Škerget, L., The numerical simulation of non-linear separation columns by boundary-domain integral formulation. Computers Chem. Engng, 19(Suppl.), pp. 785790, 1995.

[12] Qiu, Z.H., Wrobel, L.C. \& Power, H., Numerical solution of convection, diffusion problems at high Peclet number using boundary elements. Internat. J. Numer. Methods Engrg., 41, pp. 899-914, 1998.

[13] Peric, M. \& Ferziger, J.H., Computational Methods for Fluid Dynamics. SpringerVerlag: Berlin, Heidelberg, 1999.

[14] Vierendeels, J., Merci, B. \& Dick, E., Numerical study of the natural convection heat transfer with large temperature differences. Internat. J. Numer. Methods Heat Fluid Flow, 11, pp. 329-341, 2001.

[15] Wrobel, L.C., The Boundary Element Method, Vol.1, Applications in Thermo-Fluids and Acoustics. John Wiley and Sons: New York, 2002. 
[16] Samec, N. \& Škerget, L., Integral formulation of a diffusive-convective transport equation for reacting flows. Eng. Anal. Bound. Elem., 28, pp. 1055-1060, 2004.

[17] Škerget, L., Hriberšek, M. \& Žunič, Z., Natural convection flows in complex cavities by BEM. Internat. J. Numer. Methods Heat Fluid Flow, 13, pp. 720-735, 2003.

[18] Škerget, L. \& Samec, N., BEM for the two-dimensional plane compressible fluid dynamics. Eng. Anal. Bound. Elem., 29, pp. 41-57, 2005.

[19] Ramšak, M. \& Škerget, L., A multidomain boundary element method for two equation turbulence models. Eng. Anal. Bound. Elem., 29, pp. 1086-1103, 2005.

[20] Ravnik, J., Škerget, L. \& Hriberšek, M., Two-dimensional velocity-vorticity based LES for the solution of natural convection in a differentially heated enclosure by wavelet transform based BEM and FEM. Eng. Anal. Bound. Elem., 30, pp. 671-686, 2006.

[21] Dixit, H. \& Babu, V., Simulation of high Rayleigh number natural convection in a square cavity using the lattice Boltzmann method. Int. J. Heat Mass Transfer, 46, pp. 727-739, 2006.

[22] Popov, V., Power, H. \& Škerget, L., Domain decomposition techniques for boundary elements, application to fluid flow. Advances in Boundary Element Series, WIT Press: Southampton, Boston, 2007.

[23] Sajjadi, H., Gorji, M., Kefayati, G.H.R., Ganji, D.D. \& Shayan, Nia M., Numerical analysis of turbulent natural convection in a square cavity using large eddy simulation in lattice Boltzmann method. WASET, 61, pp. 819-823, 2012.

[24] Lupše, J., Škerget, L. \& Ravnik, J., Velocity-vorticity (RANS) turbulence modeling by boundary element method. Eng. Anal. Bound. Elem., 39, pp. 44-52, 2014.

[25] Kocutar, P., Škerget, L. \& Ravnik, J., Hybrid LES/URANS simulation of turbulent natural convection by BEM. Eng. Anal. Bound. Elem., 61, pp. 16-26, 2015. 